\title{
The influence of migratory background and parental education on health care utilisation of children
}

\author{
Nicole Rosenkötter • Martien C. J. M. van Dongen • \\ Wolfgang Hellmeier • Klaus Simon • Pieter C. Dagnelie
}

Received: 3 January 2012 / Accepted: 5 June 2012 /Published online: 23 June 2012

(C) The Author(s) 2012. This article is published with open access at Springerlink.com

\begin{abstract}
School-entry screening data from North RhineWestphalia (NRW), Germany from 2007 were used to investigate child health care utilisation. We focussed on the influence of migratory background and parental education on children's (1) participation in regularly scheduled early recognition examinations, (2) immunisation uptake and (3) referrals due to a school-entry screen-detected complaint. The study sample consisted of 52,171 children out of 17 NRW districts. Bivariable, stratified and multivariable analyses were performed to identify relevant associations between social determinants and health care utilisation outcome parameters. Multivariable logistic regression showed that children belonging to families with a migratory background or low parental education were more likely to have an incomplete documentation of early recognition examinations and to be referred due to a new diagnosis. Children with migratory background were more likely to be
\end{abstract}

Some results of this study were presented during the annual joint conference of the German Society for Epidemiology and the German Society for Social Medicine and Prevention in Berlin, Germany (August 22, 2010).

\footnotetext{
N. Rosenkötter $(\bowtie)$

Department of International Health, CAPHRI School for Public Health and Primary Care, Faculty of Health, Medicine and Life Sciences, Maastricht University,

Duboisdomein 30,

6229 GT, Maastricht, The Netherlands

e-mail: nicole.rosenkoetter@maastrichtuniversity.nl
}

M. C. J. M. van Dongen • P. C. Dagnelie

Department of Epidemiology, Faculty of Health,

Medicine and Life Sciences, Maastricht University,

Maastricht, The Netherlands

W. Hellmeier $\cdot$ K. Simon

Department of Health Information, NRW Centre for Health,

Bielefeld, Germany sufficiently immunised than children with parents of German ethnicity. For all studied health care utilisation outcomes, kindergarten visit had a supportive effect. Conclusion: In general, the results of our regional study were in line with the results from national populationbased studies. Additionally, a larger likelihood of referrals due to school-screen-detected deficits in children with migratory background or low parental education was detected.

Keywords Child health $\cdot$ Health service usage $\cdot$ Migration and health $\cdot$ Social differences

\section{Introduction}

Universal access to health care services is a precondition for good health [3,21]. Although it may be assumed that public social security systems, as they exist in Germany, ensure universal access to health care services, inequalities have been described in countries with well-developed social security systems $[1,5,8,10,11,18,19]$. For example, the participation rate in regular health check-up examinations during childhood which are nationally recommended in Germany and covered by the health insurance has shown to be up to $20 \%$ lower in families with a low socio-economic status compared to families with a high socio-economic status $[5,10]$. On the other hand, the immunisation status of children was less likely to be complete in families with a high socio-economic status $[10,11,19]$, which might be attributed to increased scepticism about the usefulness of vaccinations in this population sub-group [9]. Comparable relationships between socio-economic status and health care utilisation have been observed in other European countries as well [8].

In general, these findings were based on populationbased studies, like for example the German Health Interview 
and Examination Study of Children and Adolescents (KiGGS) [5,11] or the European KIDSCREEN study [1]. In addition, children's health care utilisation can be analysed with data from regular school-entry screening programmes, which are routinely conducted in most parts of Germany. Koller and Mielck have used school-entry screening data in the city of Munich to identify inequalities in children's health care utilisation [6]. Since data on social determinants, such as educational level, were not available at the individual level, Koller and Mielck used aggregated data on school district level to perform an ecological study [6].

In contrast to Koller and Mielck [6], North RhineWestphalia (NRW, Germany) school-entry screening programmes provide individual data for some social determinants. By using NRW school-entry screening data, we aimed to analyse children's health care utilisation. We intended to verify the results from the analysis of Koller and Mielck [6] and the national population-based KiGGS study $[5,11]$ regarding the influence of migratory background and social status on participation in early recognition examinations and immunisation uptake during childhood. Furthermore, we aimed to go beyond these studies by analysing the relationship between migratory background and educational level of the families and the identification of new diagnoses during school-entry screening, which might indicate a lack of previous health care utilisation.

\section{Materials and methods}

The school-entry screening programme in NRW is compulsory and regulated by law and has the aim to identify if the children are health-wise capable of starting school. For each yearly screening round, the census sample of children aged 5 to 6 is invited. The resulting data from these screening examinations were used for a secondary analysis of health service utilisation with an observational, cross-sectional study design.

The study population included all children who participated in the 2007 school-entry screening from districts of NRW that performed medical school examinations in accordance with a standardised model, the so-called Bielefeld model [7,15], and collected data on social determinants by a predefined questionnaire. The Bielefeld model is applied by approximately $80 \%$ of the local health authorities and defines the school-entry screening procedure, applies standardised paediatric definitions for diagnostic findings and provides a standardised and anonymous form for data collection.

The analysis of the occurrence of new diagnoses detected during school-entry screening included only a sub-group of children. Those with at least one diagnostic finding on visual or hearing acuity, or deficits in kinetic or cognitive development were included.

The outcome variables were related to three different domains of child health care utilisation. The first outcome variable was the level of participation of children in the nationally recommended series of nine early recognition examinations which are scheduled at birth to the age of about 5 years. For further analysis, this outcome measure was dichotomised: complete (no examination skipped) vs. incomplete (at least one examination skipped). The level of uptake of nationally recommended vaccinations during childhood was assessed as the second outcome variable. The vaccination status was regarded as sufficient when in the 'International Certificates of Vaccination' of the children nationally recommended vaccinations were documented in the necessary dose. For further analysis, this outcome was dichotomised: complete vs. incomplete (at least one vaccination missing or insufficient) immunisation uptake. Participation in both, the early recognition examinations and the immunisation programme, was assessed by investigation of the respective documentation booklets. Based on the findings of a previous study [20], it was assumed that a child had not sufficiently participated if the child's parents failed to hand over the relevant booklet.

Referral (yes/no) to specialist care due to a new schoolentry screen-detected diagnosis was the third outcome measure. If at least one complaint (decreased visual or hearing acuity, or deficits in kinetic or cognitive development) had been recorded during the school-entry screening, we distinguished between children who were already under treatment for this complaint, indicating that they had gained access to the health care system in the meantime, and children who were referred to a practitioner for further diagnosis and treatment, indicating that health care utilisation might have been deficient previously. A referral could occur in children who had never been diagnosed before with this complaint, but also in children who had been diagnosed earlier, for instance with decreased visual acuity for which the current glasses were not sufficient anymore. In the following, this outcome is shortly named 'referral'.

Parental education is one of the primary independent variables. It was constructed by combining information on the highest educational grade and the highest training qualifications of either the child's father or mother [4]. For further analysis, this score was trichotomised: scores 1 to 3 representing a low parental education, scores 4 to 6 medium parental education and scores 7 to 8 high parental education.

To gain information on the migratory background, the other primary independent variable, parents were asked which language they mainly speak at home with the child. The questionnaire differentiated between 'German' and 'other language'. Since 'other language' was not further specified, no information on the cultural background was 
available. When a language other than German was spoken with the child, a migratory background was assumed. Actually, this variable reflected the level of integration of the families in German society rather than the concept of migratory background as it is commonly used in official population statistics [13].

Independent variables of secondary interest were single parent household (yes/no), presence of siblings (yes/no), duration of kindergarten attendance (at least vs. less than 12 months) and urbanisation degree of living area (rural/ urban). Larger cities are defined in NRW as independent urban municipalities (like Cologne, Düsseldorf, etc.), and smaller cities are compiled with rural surroundings to rural districts. This official distinction of NRW regions was used to define the urbanisation degree of the living area of the children. NRW consisted (in 2007) officially of 23 urban municipalities and 31 rather rural districts. Furthermore, the participation in early recognition examinations was used as an additional determinant in the analysis of immunisation uptake and referrals. Since children were often vaccinated during early recognition examinations and comparable tests, e.g., visual or audiometry tests were performed during early recognition examinations and the school-entry screening, an influence on each of the two outcomes can be hypothesised. As a consequence, the level of participation in early recognition examinations might act predominately as the intermediate variable in the causal pathways linking the determinants of primary interest to the outcomes of immunisation uptake and referral. Our main interest was in assessing the residual effect of migratory background and parental education remaining after adjustment for participation in early recognition examinations.

For descriptive purposes, absolute and relative frequencies were calculated for all dependent and independent variables. To estimate the strength of the associations between the primary determinants and each of the outcome variables, crude odds ratios (OR) were calculated based on simple cross-table analyses. Subsequently, based on stratified cross-table analyses, Mantel-Haenszel odds ratios $\left(\mathrm{OR}_{\mathrm{MH}}\right)$ were calculated for the primary determinants, taking the effects of other single determinants into account. Finally, multiple logistic regression analyses were performed to adjust the associations between the primary determinants and each outcome variable for the combined influence of multiple covariates.

For each multiple logistic regression analysis, the initial model was built by entering all predictors that seemed to be relevant based on the literature and the results of the crosstable analyses. Each model was tested beforehand for multicollinearity of the included predictors, which resulted to be absent. For each outcome variable, the final model was constructed by backward elimination of covariates. Missing values were treated by case-wise deletion. Interaction effects were presented by showing the main effects. All statistical analyses were performed with SPSS 15.01 (SPSS Inc., Chicago, IL, USA).

\section{Results}

In $2007,168,389$ children from 54 districts participated in the school-entry screening in NRW. A total number of 116,218 children had to be excluded from data analysis, for various reasons: (1) living in districts in which the Bielefeld model for school entry screening was not applied $(n=8,924)$; (2) living in districts in which information on social determinants was not collected consistently $(n=$ 105,770); (3) high potential for coding error for the age variable $(n=402)$; and (4) living in a district with potential coding errors for several variables $(n=1,122$; coding errors occurred for the educational level of the parents and for new school-entry screen-detected complaints). Therefore, the final sample eligible for analysis consisted of 52,171 children ( $31 \%$ of the initial sample) from 17 districts in NRW. The children originated equally from rural $(n=25,529 ; 48.9 \%)$ and urban $(n=26,642 ; 51.9 \%)$ districts. Since NRW is a diverse federal state in Germany, the included districts are further characterised by clusters which share sociodemographic characteristics, and the distribution of districts was made according to an updated analysis in $2011[16,17]$. In the study sample, regions from all occurring clusters in NRW are included: less family-defined university cities (one of four districts in NRW), poor cities and districts in structural change ( 8 of 16 districts in NRW); wealthy shrinking and ageing cities and sub-urban regions (one of six districts in NRW); growing family zones (4 of 15 districts in NRW), growing and prospering regions (one of six districts in NRW); and family-defined districts with a tendency of declining and ageing (two of seven districts in NRW). The age of the children ranged from 61 to 82 months, and the sample comprised 26,867 boys (51.5\%) and 25,304 girls (48.5\%).

Table 1 shows the absolute frequencies and prevalence rates of the unfavourable outcomes, according to the levels of each determinant. The table indicates also the available sample sizes for each determinant. The highest amount of missing values was present for the primary independent variable parental education (20.1\%), followed by the secondary independent variables siblings $(19.8 \%)$ and single parent households $(14.5 \%)$. All other variables had less than $10 \%$ missing values. These rates of missing values were slightly higher in the population sub-sample available for the analysis of determinants of the outcome referral due to a screen-detected diagnosis.

Incomplete participation in early recognition examinations (IPERE) was less prevalent (31.6\%) than incomplete immunisation uptake (IIU) $(58.7 \%)$ or referral $(55.3 \%)$ 
Table 1 Absolute numbers and prevalence (percent; $95 \% \mathrm{CI}$ ) of independent variables for the outcomes incomplete participation in early recognition examinations, incomplete immunisation uptake and referral due to at least one new diagnosis during school-entry screening NRW, 2007

All school-screened children ${ }^{\mathrm{a}}$

Independent variables

Incomplete participation in early recognition examinations (IPERE) ${ }^{\mathrm{b}}$ $n ; \%(95 \% \mathrm{CI})$
Incomplete immunisation uptake (IIU) ${ }^{\mathrm{c}}$ $n ; \%(95 \% \mathrm{CI})$

Sub-population with at least one diagnosis $^{\mathrm{d}}$

\begin{tabular}{|c|c|}
\hline $\begin{array}{l}\text { Referral }^{\mathrm{e}} \\
n ; \%(95 \% \mathrm{CI})\end{array}$ & Total \\
\hline
\end{tabular}

Total $(N=52,171)$

Migratory background $^{\mathrm{f}}(N=49,469)$

Yes

No

Parental education $^{\mathrm{g}}(N=41,697)$

Low

Medium

High

Siblings $(N=41,849)$

Yes

No

Kindergarten visit $^{\mathrm{h}}(N=49,635)$

Yes

No

Single parent household $(N=44,592)$

Yes

No

Area type $^{\mathrm{i}}(N=52,171)$

Rural

Urban

Participation in all $\mathrm{U}$ examinations $(N=52,171)$

Complete

Incomplete

$$
\begin{array}{r}
16,510 ; 31.6(31.2-32.0) \\
15,664 ; 31.7(31.3-32.1) \\
7,116 ; 53.5(52.7-54.3) \\
8,548 ; 23.6(23.2-24.0) \\
11,701 ; 28.1(27.7-28.5) \\
4,901 ; 45.5(44.5-46.3) \\
4,646 ; 22.3(21.7-22.9) \\
2,154 ; 20,7(19.9-21.5) \\
13,166 ; 31.5(31.1-31.9) \\
10,612 ; 32.2(31.7-32.7) \\
2,554 ; 28.8(27.9-29.7) \\
15,465 ; 31.2(30.8-31.6) \\
14,128 ; 29.7(29.3-30.1) \\
1,337 ; 64.5(62.4-66.6) \\
13,251 ; 29.7(29.3-30.1) \\
2,369 ; 43.5(42.2-44.8) \\
10,882 ; 27.8(27.4-28.2) \\
16,510 ; 31.6(31.2-32.0) \\
7,066 ; 27.6(27.1-28.1) \\
9,444 ; 35.4(34.8-36.0)
\end{array}
$$

${ }^{a}$ Children from the NRW districts Aachen, Bielefeld, Coesfeld, Dortmund, Essen, Euskirchen, Gelsenkirchen, Herne, Heinsberg, HochsauerlandKreis, Höxter, Krefeld, Leverkusen, Mönchengladbach, Oelde, Paderborn and Recklinghausen

${ }^{\mathrm{b}}$ At least one out of nine $U$ examinations missing

${ }^{\mathrm{c}}$ At least one out of nine vaccinations missing

${ }^{\mathrm{d}}$ Children with at least one diagnosis on visual or hearing acuity or deficits in kinetic or cognitive development

${ }^{\mathrm{e}}$ Occurrence of referral of a child due to a new diagnosis during school-entry screening

${ }^{\mathrm{f}}$ Migratory background was assumed when it was mainly not spoken German with the child at home

${ }^{\mathrm{g}}$ Summary measure of the highest school grade and training qualification of child's mother or father

${ }^{\mathrm{h}}$ At least 1 year of kindergarten visit

${ }^{\mathrm{i}}$ The area type was determined by the district of living (rural district $=$ rural, city district $=$ urban)

${ }^{\mathrm{j}}$ Participation in all $\mathrm{U}$ examinations was used as predictor variable for the outcomes of incomplete immunisation uptake and occurrence of referral due to a new diagnosis

(Table 1). Children of families with a migratory background or low parental education had substantially more often an incomplete history of early recognition examinations (53.5 and $45.5 \%$, respectively). These children were also more often referred to specialist health care (71.0 and $62.7 \%$, respectively). Compared with the reference groups, the prevalence of IIU was only slightly increased in these subgroups, however (60.9 and $61.7 \%$, respectively). Moreover, children from a single parent household and children who did not visit kindergarten were clearly more often at risk for IPERE and IIU and were more often referred for a screendetected diagnosis. Finally, IIU and referral occurred more 
frequently in children who did not complete the programme of early recognition examinations (Table 1).

Stratified cross-table analysis was conducted to assess the influence of the determinants of primary interest per stratum of each of the other independent variables. Complete participation in early recognition examinations, which was also evaluated as a determinant of the two other outcome measures, appeared to be the only independent variable responsible for a substantial shift $(>10 \%)$ of the adjusted $\mathrm{OR}_{\mathrm{MH}}$ compared with the crude OR estimate (Table 2). Stratification for participation in early recognition examinations caused a change of the strength of the summary association between migratory background and IIU from 1.13 (95\% CI $1.08-1.17$ ) to 0.91 (95\% CI 0.87-0.95) and between low parental education and IIU from 1.29 (95 \% CI 1.22-1.36) to 1.09 (95\% CI 1.03-1.16). Likewise, stratification for the same variable reduced the strength of the summary association between migratory background and referral from 2.47 (95\% CI 2.27-2.68) to 2.08 (95 \% CI 1.91-2.26) and between low parental education and referral from 1.71 (95\% CI 1.53-1.91) to 1.44 (95\% CI 1.28-1.61). The independent variable single parent household appeared as an interaction effect for the relationship between the determinants of primary interest and the outcome IPERE (Table 2). As part of the multivariable logistic regression analyses, this was further investigated, the results are presented in Table 3 .

All measured determinants were included in the model. The determinant 'siblings' appeared not to improve the model on IIU and referral and was therefore excluded. Remarkably, when taking the other independent variables into account, the association between migratory background and IIU of the child changed from $\mathrm{OR}=1.13$ (unadjusted; $95 \%$ CI 1.08-1.17) to OR=0.88 (adjusted; $95 \%$ CI 0.84 0.93 ), indicating a protective effect. Furthermore, child's visit of a kindergarten was quite strongly reversely associated with a history of IPERE $(\mathrm{OR}=3.43)$ and to a lesser degree with the other indicators of insufficient health care utilisation. In addition, living in an urban area slightly decreased the odds of being under vaccinated $(\mathrm{OR}=0.85$; $95 \%$ CI $0.81-0.88$ ), but slightly increased the probability of the other unfavourable health care utilisation outcomes.

As already indicated in the stratified analysis, the preferred model describing the association between social
Table 2 Relevant results of the stratified analysis, school-entry screening NRW, 2007
IIU incomplete immunisation uptake, IPERE incomplete participation in early recognition examinations

\begin{tabular}{|c|c|c|c|c|}
\hline & & OR $(95 \% \mathrm{CI})$ & $\begin{array}{l}\mathrm{OR}_{\mathrm{MH}} \\
(95 \% \mathrm{CI})\end{array}$ & $\begin{array}{l}\text { Breslow \& Day } \\
\text { heterogeneity test }(p)\end{array}$ \\
\hline \multicolumn{5}{|l|}{$\begin{array}{l}\text { Migratory background- } \\
\text { IIU }\end{array}$} \\
\hline Crude effect & & $1.13(1.08-1.17)$ & & \\
\hline $\begin{array}{l}\text { Participation in early } \\
\text { recognition examinations }\end{array}$ & $\begin{array}{l}\text { Complete } \\
\text { Incomplete }\end{array}$ & $\begin{array}{l}0.91(0.86-0.96) \\
0.91(0.85-0.98)\end{array}$ & $0.91(0.87-0.95)$ & 0.872 \\
\hline \multicolumn{5}{|l|}{$\begin{array}{l}\text { Low parental education- } \\
\text { IIU }\end{array}$} \\
\hline Crude effect & & $1.29(1.22-1.36)$ & & \\
\hline $\begin{array}{l}\text { Participation in early } \\
\text { recognition examinations }\end{array}$ & $\begin{array}{l}\text { Complete } \\
\text { Incomplete }\end{array}$ & $\begin{array}{l}1.12(1.05-1.20 \\
1.01(0.90-1.13)\end{array}$ & $1.09(1.03-1.16)$ & 0.098 \\
\hline \multicolumn{5}{|l|}{$\begin{array}{l}\text { Migratory background- } \\
\text { referral }\end{array}$} \\
\hline Crude effect & & $2.47(2.27-2.68)$ & & \\
\hline $\begin{array}{l}\text { Participation in early } \\
\text { recognition examinations }\end{array}$ & $\begin{array}{l}\text { Complete } \\
\text { Incomplete }\end{array}$ & $\begin{array}{l}2.03(1.82-2.27) \\
2.14(1.88-2.44)\end{array}$ & $2.08(1.91-2.26)$ & 0.552 \\
\hline \multicolumn{5}{|l|}{$\begin{array}{l}\text { Low parental education- } \\
\text { referral }\end{array}$} \\
\hline Crude effect & & $1.71(1.53-1.91)$ & & \\
\hline $\begin{array}{l}\text { Participation in early } \\
\text { recognition examinations }\end{array}$ & $\begin{array}{l}\text { Complete } \\
\text { Incomplete }\end{array}$ & $\begin{array}{l}1.40(1.22-1.60) \\
1.55(1.25-1.94)\end{array}$ & $1.44(1.28-1.61)$ & 0.438 \\
\hline \multicolumn{5}{|c|}{ Migratory background-IPERE } \\
\hline Crude effect & & $3.71(3.56-3.87)$ & & \\
\hline Single parent household & $\begin{array}{l}\text { No } \\
\text { Yes }\end{array}$ & $\begin{array}{l}4.09(3.89-4.30) \\
2.74(2.36-3.17)\end{array}$ & $3.91(3.73-4.10)$ & $<0.001$ \\
\hline
\end{tabular}


Table 3 Results of multivariable analysis (logistic regression), school-entry screening NRW, 2007

\footnotetext{
aAdjusted for kindergarten visit, area type, siblings, migratory background or parental education, respectively. Interaction term for migratory background and single parent household was included in the final model

${ }^{\mathrm{b}}$ Adjusted for single parent household, kindergarten visit, area type, participation in early recognition examinations, migratory background or parental education, respectively

${ }^{\mathrm{c}}$ Adjusted for single parent household, kindergarten visit, area type, participation in early recognition examinations, migratory background or parental education, respectively
}

\begin{tabular}{|c|c|c|c|c|c|c|}
\hline & \multicolumn{2}{|c|}{$\begin{array}{l}\text { Incomplete participation in early } \\
\text { recognition examinations (IPERE) }\end{array}$} & \multicolumn{3}{|c|}{$\begin{array}{l}\text { Incomplete immunisation } \\
\text { uptake }(\mathrm{IIU})^{\mathrm{b}}\end{array}$} & \multirow{2}{*}{$\begin{array}{l}\text { Referral }^{\mathrm{c}} \\
95 \% \mathrm{CI}\end{array}$} \\
\hline & OR & $95 \%$ CI & OR & $95 \% \mathrm{CI}$ & OR & \\
\hline \multicolumn{7}{|l|}{ Migratory background } \\
\hline Yes & - & - & 0.88 & $0.84-0.93$ & 1.85 & $1.66-2.06$ \\
\hline No & - & - & 1 & & 1 & \\
\hline \multicolumn{7}{|l|}{ Parental education } \\
\hline Low & 2.04 & $1.89-2.20$ & 1.12 & $1.05-1.19$ & 1.26 & $1.12-1.42$ \\
\hline Medium & 1.05 & $0.98-1.13$ & 1.04 & $0.98-1.09$ & 0.95 & $0.86-1.06$ \\
\hline High & 1 & & 1 & & 1 & \\
\hline \multicolumn{7}{|l|}{ Siblings } \\
\hline Yes & 1.25 & $1.16-1.34$ & - & - & - & - \\
\hline No & 1 & & - & - & - & - \\
\hline \multicolumn{7}{|l|}{ Kindergarten visit } \\
\hline No & 3.43 & $2.97-3.97$ & 1.27 & $1.11-1.44$ & 1.77 & $1.39-2.25$ \\
\hline Yes & 1 & & 1 & & 1 & \\
\hline \multicolumn{7}{|l|}{ Single parent household } \\
\hline Yes & & & 1.09 & $1.02-1.17$ & 1.29 & $1.14-1.46$ \\
\hline No & & & 1 & & 1 & \\
\hline \multicolumn{7}{|l|}{ Area type } \\
\hline Urban & 1.21 & $1.15-1.28$ & 0.85 & $0.81-0.88$ & 1.15 & $1.06-1.25$ \\
\hline Rural & 1 & & 1 & & 1 & \\
\hline \multicolumn{7}{|l|}{ Participation in $U$ examinations } \\
\hline Incomplete & - & - & 1.91 & $1.82-2.01$ & 1.64 & $1.49-1.81$ \\
\hline Complete & - & - & 1 & & 1 & \\
\hline \multicolumn{7}{|l|}{ Interaction } \\
\hline \multicolumn{7}{|l|}{ migratory background } \\
\hline Single parent household & 2.07 & $1.61-2.68$ & & & & \\
\hline Non-single parent household & 3.10 & $2.91-3.95$ & & & & \\
\hline
\end{tabular}

determinants and participation in early recognition examinations appeared to include a term reflecting the interactive effect of migratory background and growing up in a single parent household. Therefore, the association between migratory background and participation in early recognition examinations was assessed separately for single parent households $(\mathrm{OR}=2.07 ; 95 \% \mathrm{CI} 1.61-2.68)$ and non-single parent households $(\mathrm{OR}=3.10,95 \% \mathrm{CI} 2.91-3.95)$.

\section{Discussion}

Our study results showed an increased likelihood of IPERE for children of families with a low educational index and with a migratory background. After adjustment for other risk factors, not visiting the kindergarten showed the strongest association in children with IPERE. Children with a migratory background were more likely to be sufficiently vaccinated compared to children without such a background. Moreover, an increased likelihood of referrals was observed for these children and children with low parental education, even after adjustment for participation in early recognition examinations. Again, kindergarten visit seems to have a positive influence on the timely and adequate identification and treatment of complaints like visual or hearing acuity and kinetic or cognitive development.

Generally, our study results on IPERE and IIU by children from the included districts in NRW are in line with previous findings from the national KiGGS study $[5,11]$ and with outcomes of the analyses of Koller and Mielck on school district level [6]. The prevalence rate of IPERE in children with migratory background in the current study was comparable with the prevalence rate observed in the KiGGS study for children with parents having both a migratory background [14]. This observation is compatible, since our definition of migratory background was based on the parents' report not to speak German with their child at home, which is more likely when both parents have a migratory background. 
Another often described observation is the increased likelihood of IIU in children of families with high socioeconomic status $[11,12,19]$, which was not found in our analysis. Only a descriptive analysis of the participation in separate vaccinations showed that the prevalence of IIU in these children was often as high or even higher than for children of families with low parental education (results were not shown). However, when analysing the completeness of the combination of all nine vaccinations, children of families with high parental education seemed to have more often all vaccinations complete than children of families with low parental education.

The interactive effect of migratory background and single parent household on the participation in early recognition examinations seems to be misleading since this interaction resulted unexpectedly in a smaller effect for children with migratory background living in single parent households $(\mathrm{OR}=2.07)$ compared to children with migratory background living in non-single parent households $(\mathrm{OR}=3.10)$. This observation originates from the fact that unless the respective prevalence of IPERE is higher in children from single parent households (migratory background $63.8 \%$, non-migratory background $39.2 \%$ ) compared to children from non-single parent households (migratory background $50.5 \%$, non-migratory background $19.9 \%$ ), the difference in prevalence between children with migratory background and non-migratory background is larger in children from non-single parent households (difference $30.6 \%$ points) compared to children from single parent households (difference $24.6 \%$ points).

Due to the cross-sectional design and the use of secondary data, our study has several limitations which might have led probably to an overestimation of the strength of the associations. We were not able to determine, for instance, if the screen-detected complaints, resulting in the referral of the child, originated months or years ago and thus could have been detected earlier, or if they arose recently. In addition, there was neither information on the severity of the complaints available, nor if the diagnosis of the school-entry screening was subsequently confirmed by a practitioner or specialist. Moreover, due to migration during the life course of the child, the documentation booklets could have been incomplete. It was not possible to adjust the results accordingly.

Furthermore, we were not able to analyse if insufficient health care utilisation was related to large distances to the next health care provider. This influence was only roughly taken into account by the inclusion of the type of the living area as one determinant for health service use.

The high proportion of missing values for the variable parental education could have affected the results as well. Therefore, we performed a sensitivity analysis using two scenarios, in which all missing values for this variable were imputed by the highest (best case) and the lowest value (worst case), respectively. It turned out that the worst case scenario, which was probably the most realistic one since missing values for other variables occurred mostly in children with a low parental education, hardly led to any changes in the observed associations (results not shown).

In this study, we focussed on the health care utilisation of preventive services (early recognition examinations, immunisation). Also the outcome measure 'referral' concentrated just on deficits of visual or hearing acuity or kinetic or cognitive development, and not on any acute disorders. Therefore, it was not possible to make general assumptions on health care utilisation of children of families with migratory background or low parental education. National results on the use of ambulant health care services of pre-scholars showed for example no differences in the use of paediatricians and GPs between migrants and non-migrants and children from different social status. Only the use of specialist services, as it is required after referral due to a school screen-detected diagnosis in the current study, was different nationally. A less frequent use of specialists, especially ophthalmologists, in migrants and children of low social status was observed [5].

Another interesting observation was the fact that not visiting the kindergarten was strongly associated with a lack of health care utilisation, as it was measured in this study. This underlines the importance of kindergartens not only for the individual development of the child [2] but also as an assisting institution for families with regard to the use of preventive and health care services.

In conclusion, we could show that children's health care service use is influenced by social determinants. Regional data from the NRW school-entry screening programme proved to be a useful, yearly available data source for research on health service use. In order to gain a deeper understanding on barriers that hinder equal health care utilisation and to be able to improve health services accordingly, these data could be an appropriate source for extended analyses.

Conflict of interest The authors declare that they have no conflict of interest and received no financial support to perform this analysis.

Open Access This article is distributed under the terms of the Creative Commons Attribution License which permits any use, distribution, and reproduction in any medium, provided the original author(s) and the source are credited.

\section{References}

1. Berra S, Tebe C, Erhart M, Ravens-Sieberer U, Auquier P, Detmar S, Herdman M, Alonso J, Rajmil L (2009) Correlates of use of health care services by children and adolescents from 11 European countries. Med Care 47(2):161-167. doi:10.1097/MLR.0b013e3181844e09 
2. Caniato RN, Alvarenga ME, Stich HL, Jansen H, Baune BT (2010) Kindergarten attendance may reduce developmental impairments in children: results from the Bavarian Pre-School Morbidity Survey. Scand J Public Health 38(6):580-586. doi:10.1177/ 1403494810376558

3. Dahlgren G, Whitehead M (1992) Policies and strategies to promote social equity in health. Background document to WHO. Strategies for Europe. Institute of Future Studies, Stockholm. Available at: http://www.framtidsstudier.se/wp-content/uploads/ 2011/01/20080109110739filmZ8UVQv2wQFShMRF6cuT.pdf. Accessed July 2010

4. Jöckel KH, Babitsch B, Bellach BM, Bloomfield K, HoffmeyerZlotnik J, Winkler J, Wolf C (1998) Messung und Quantifizierung soziographischer Merkmale in epidemiologischen Studien. Available at: http://www.rki.de/cln 100/nn 205212/DE/Content/GBE/ EpidemiologischeMethoden/Empfehlungen/empfehlungen pdf2, templateId=raw, property=publicationFile.pdf/empfehlungen pdf2.pdf. Accessed July 2010

5. Kamtsiuris P, Bergmann E, Rattay P, Schlaud M (2007) Inanspruchnahme medizinischer Leistungen. Ergebnisse des Kinderund Jugendgesundheitssurveys (KIGGS). Bundesgesundheitsbl Gesundheitsforsch Gesundheitsschutz 50(5-6):836-850. doi:10.1007/s00103-007-0247-1

6. Koller D, Mielck A (2009) Regional and social differences concerning overweight, participation in health check-ups and vaccination. Analysis of data from a whole birth cohort of 6-year old children in a prosperous German city. BMC Publ Health 9:43. doi:10.1186/1471-2458-9-43

7. Gesundheit NRW (2008) Das Bielefelder Modell zur Schuluntersuchung. Available at: http://www.lzg.gc.nrw.de/themen/ gesundheit_berichte_daten/schulgesundheit/bielefelder-modell/ index.html. Accessed March 2012

8. Majeed M, Williams C, Northstone K, Ben-Shlomo Y (2008) Are there inequities in the utilisation of childhood eye-care services in relation to socio-economic status? Evidence from the ALSPAC cohort. Br J Ophthalmol 92(7):965-969. doi:10.1136/bjo.2007.134841

9. Meyer C, Reiter S (2004) Impfgegner und Impfskeptiker. Geschichte, Hintergründe, Thesen, Umgang. Bundesgesundheitsbl Gesundheitsforsch Gesundheitsschutz 47(12):1182-1188

10. Oberwöhrmann S, Bettge S (2007) Basisdaten zur gesundheitlichen und sozialen Lage von Kindern in Berlin - Ergebnisse auf der Basis der Einschulungsuntersuchungen 2005. Gesundheitsberichterstattung Berlin, Spezialbericht 2007 - 1. Senatsverwaltung für Gesundheit, Umwelt und Verbraucherschutz, Berlin
11. Poethko-Muller C, Kuhnert R, Schlaud M (2007) Durchimpfung und Determinanten des Impfstatus in Deutschland. Ergebnisse des Kinder- und Jugendgesundheitssurveys (KIGGS). Bundesgesundheitsbl Gesundheitsforsch Gesundheitsschutz 50(5-6):851-862

12. Poethko-Muller C, Ellert U, Kuhnert R, Neuhauser H, Schlaud M, Schenk L (2009) Vaccination coverage against measles in Germanborn and foreign-born children and identification of unvaccinated subgroups in Germany. Vaccine 27(19):2563-2569

13. Schenk L, Bau AM, Borde T, Butler J, Lampert T, Neuhauser H, Razum O, Weilandt C (2006) Mindestindikatorensatz zur Erfassung des Migrationsstatus. Empfehlungen für die epidemiologische Praxis. Bundesgesundheitsbl Gesundheitsforsch Gesundheitsschutz 49 (9):853-860. doi:10.1007/s00103-006-0018-4

14. Schenk L, Neuheuser H, Ellert U, Poethko-Müller C, Kleiser C, G. M (2008) Kinder- und Jugendgesundheitssurvey (KiGGS) 20032006: Kinder und Jugendliche mit Migrationshintergrund in Deutschland. Gesundheitsberichterstattung des Bundes, Berlin

15. Schirm H (1989) Untersuchungen und Untersuchungsmodelle zur Feststellung der Einschulungsfähigkeit. Sozial Pädagogik: 7-11

16. Schultz A (2011) Aktualisierung der Clusteranalyse mit Sozialstruktur-indikatoren für Kreise und kreisfreie Städte in NRW - Gesundheitsberichterstattung Nordrhein-Westfalen - 2005 und 2008. Faktor Familie GmbH, Bochum

17. Strohmeier KP, Schultz A, Bardehle D, Annuss R, Lenz A (2007) Health indicator-based cluster analysis of districts and urban districts in North Rhine-Westphalia. Gesundheitswesen 69(1):26-33. doi:10.1055/s-2007-960491

18. von Rueden U, Gosch A, Rajmil L, Bisegger C, Ravens-Sieberer U (2006) Socioeconomic determinants of health related quality of life in childhood and adolescence: results from a European study. J Epidemiol Community Health 60(2):130-135. doi:10.1136/ jech.2005.039792

19. Wei F, Mullooly JP, Goodman M, McCarty MC, Hanson AM, Crane B, Nordin JD (2009) Identification and characteristics of vaccine refusers. BMC Pediatr 9:18. doi:10.1186/14712431-9-18

20. Wichmann O, Hellenbrand W, Sagebiel D, Santibanez S, Ahlemeyer G, Vogt G, Siedler A, van Treeck U (2007) Large measles outbreak at a German public school, 2006. Pediatr Infect Dis J 26(9):782-786. doi:10.1097/INF.0b013e318060aca1

21. World Health Organisation The determinants of health. Available at: http://www.who.int/hia/evidence/doh/en/print.html. Accessed July 2010 